\title{
Critical Boundary of Cascaded Quadratic Soliton Compression in PPLN
}

\author{
Guo, Hairun; Zeng, Xianglong; Zhou, Binbin; Bache, Morten
}

Published in:

CLEO: Applications and Technology, CLEO_AT 2012

Publication date:

2012

Document Version

Publisher's PDF, also known as Version of record

Link back to DTU Orbit

Citation (APA):

Guo, H., Zeng, X., Zhou, B., \& Bache, M. (2012). Critical Boundary of Cascaded Quadratic Soliton Compression in PPLN. In CLEO: Applications and Technology, CLEO_AT 2012 (pp. JW4A.45). Optical Society of America.

\section{General rights}

Copyright and moral rights for the publications made accessible in the public portal are retained by the authors and/or other copyright owners and it is a condition of accessing publications that users recognise and abide by the legal requirements associated with these rights.

- Users may download and print one copy of any publication from the public portal for the purpose of private study or research.

- You may not further distribute the material or use it for any profit-making activity or commercial gain

- You may freely distribute the URL identifying the publication in the public portal

If you believe that this document breaches copyright please contact us providing details, and we will remove access to the work immediately and investigate your claim 


\title{
Critical Boundary of Cascaded Quadratic Soliton Compression in PPLN
}

\author{
Hairun Guo*, Xianglong Zeng, Binbin Zhou and Morten Bache \\ DTU Fotonik, Department of Photonics Engineering, Technical University of Denmark, DK-2800 Kgs. Lyngby, Denmark \\ *haig@fotonik.dtu.dk
}

\begin{abstract}
Cascaded quadratic soliton compression in PPLN is investigated and a general critical soliton number is found as the compression boundary. An optimal-parameter diagram for compression at $1550 \mathrm{~nm}$ is presented.

OCIS codes: (190.4360) Nonlinear optics, devices; (190.7110) Ultra fast nonlinear optics.
\end{abstract}

\section{Introduction}

Soliton pulse compression based on cascaded quadratic nonlinearity is attractive because few-cycle near-IR pulses can be formed in short nonlinear crystals. The basic principle is that a self-defocusing cascading nonlinearity $n_{2, \text { casc }} \propto d_{\text {eff }}^{2} / \Delta k$ counterbalances the native Kerr self-focusing nonlinearity $n_{2, \text { Kerr }}$ and through spectral broadening from self-phase modulation (SPM) a temporal soliton can form with normal dispersion that compresses the pulse in the initial soliton formation stage.

A systematical study on how the cascaded quadratic nonlinearity works in pulse compression in bulk $\beta$-barium borate (BBO) has already been reported [1-3]. An effective soliton number $N_{\text {eff }} \propto\left(n_{2, \text { casc }}+n_{2, \text { Kerr }}\right)$ was introduced to represent the counterbalance between the cascaded quadratic nonlinearity and the native self-focusing cubic nonlinearity. A successful pulse compression is supported by a $N_{\text {eff }}$ larger than a critical value, which is unity for low intensities but increases for higher intensities due to cross-phase modulation (XPM) contributions to the nonlinear phase shift [4]. In this paper, we will further investigate cascaded quadratic soliton compression in a lithium niobate (LN) nonlinear crystal where the phase mismatch is controlled with quasi phase matching (QPM) structure. Using QPM is attractive as it exploits the largest quadratic nonlinearity $d_{e f f}$, while with QPM a reduction on the phase mismatch $\Delta k$ will help increasing the cascaded quadratic nonlinearity. Moreover, we find a general critical value of $N_{\text {eff }}$ for periodically poled LN (PPLN).

\section{Compression window for PPLN}

We use PPLN as it is the most common material with QPM structure. In a Type-0 phase-matching configuration, the $2^{\text {nd }}$ order nonlinearity mainly stems from the large $d_{33}$ tensor component. The cost of using QPM is an extra prefactor $2 / \pi$ due to the $1^{\text {st }}$ order QPM structure, i.e. $d_{\text {eff }}=(2 / \pi) \cdot d_{33}$. Besides, The QPM changes the phase mismatch to an effective phase mismatch, $\Delta k_{\text {eff }}=\Delta k-2 \pi / \Lambda$. In past studies QPM cascading was used to generate the largest possible cascaded nonlinearity by choosing $\Delta k_{\text {eff }} L=\pi$. However, this is not optimal for ultrafast cascading: Firstly, $\Delta k_{\text {eff }}$ should remain large to ensure a Kerr-like cascading (where the nonlinear index change is linear in intensity $\Delta n \propto n_{2, \text { casc }} I$ ), but small enough to compensate for the Kerr nonlinearity. Secondly, if $\Delta k_{\text {eff }}$ becomes too small, the cascading becomes nonstationary (resonant) and for ultrafast compression a stationary (non-resonant) nonlinearity is required. The $\Delta k_{\text {eff }}$ operation regime where this is possible is usually called compression window [2].

\section{Results and discussion}
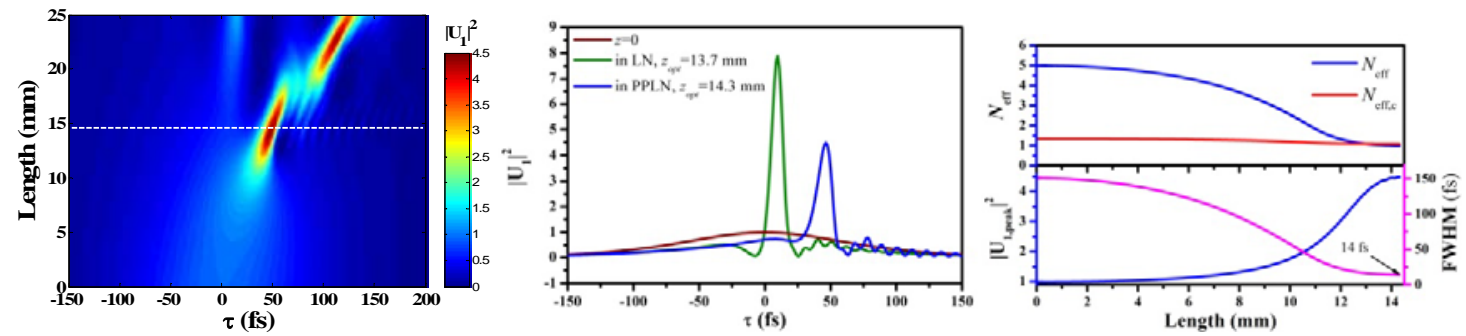

Fig. 1. (a, left) Temporal evolution of the pulse propagation and compression in a PPLN with $N_{\text {eff }}=5$, (b, middle) pulse intensity profile at the best compressed length $\mathrm{z}=14.3 \mathrm{~mm}$, (c, right) the dynamics of soliton number as well as the critical soliton number during the compression process. 
We investigate the pulse compression in PPLN at wavelength $1550 \mathrm{~nm}$. The phase mismatch of bulk LN at this wavelength is $\Delta k=319.5 \mathrm{~mm}^{-1}$. In PPLN with a poling period of $33 \mu \mathrm{m}$, the effective phase mismatch is $\Delta k_{\text {eff }}=$ $129.5 \mathrm{~mm}^{-1}$. The stationary threshold is $\Delta k_{s r}=113.1 \mathrm{~mm}^{-1}$. The reduction of the phase mismatch happens to compensate the loss on $d_{33}$ and therefore the value of self-defocusing cascading nonlinearity is as same as what in bulk LN, $n_{2, \text { casc }}=-2.8 \times 10^{-15} \mathrm{~cm}^{2} / \mathrm{W}$, which in quantity is higher than the native Kerr self-focusing nonlinearity $n_{2, \text { Kerr }}$ $=1.7 \times 10^{-15} \mathrm{~cm}^{2} / \mathrm{W}$. Hence, $\Delta k_{\text {eff }}$ is exactly in the compression window. Using a pulse with $150 \mathrm{fs}$ FWHM and 75 $\mathrm{GW} / \mathrm{cm}^{2}$ peak intensity, $N_{\text {eff }}=5$ is achieved and simulation shows that few-cycle pulse is generated after a $14.3 \mathrm{~mm}$ propagation, see Fig. 1. The compressed pulse has a FWHM of 14 fs (no more than 3 cycles) and the peak intensity is increased over 4 times. Compared with the soliton pulse generated in bulk LN (10.1 fs and 8 times in intensity), the soliton pulse generated in PPLN is a little degraded with lower peak intensity and longer pulse duration. This degradation is mainly due to the high level of GVM which sets a high stationary threshold. Then the QPM-induced reduction of phase mismatch forces the compression to be close to the stationary boundary and therefore to be less clean. In the contrary, if GVM is reduced, $\Delta k_{\text {eff }}$ will have enough space of reduction. Then a higher level of $n_{2, \text { casc }}$ in PPLN than that in bulk LN is expectable and the compression will be upgraded.

Another way to supply the cascading nonlinearity is to increase the energy fluence, which is defined as $\Phi=$ $2 T_{0} I_{0}$. Since the high energy fluence will evoke a significant Kerr XPM, which adds to the Kerr SPM and thereby dynamically increases the overall competing Kerr self-focusing nonlinearity, the compression condition $N_{\text {eff }}>1$ will be no longer enough. A critical effective soliton number $N_{e f f, c}$ must be found and the pulse compression is therefore

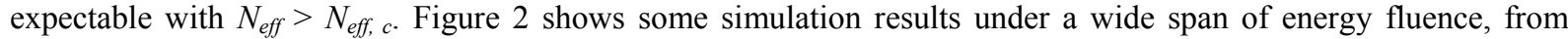
which a boundary of $N_{\text {eff }}$ is found as $N_{\text {eff, }}$.
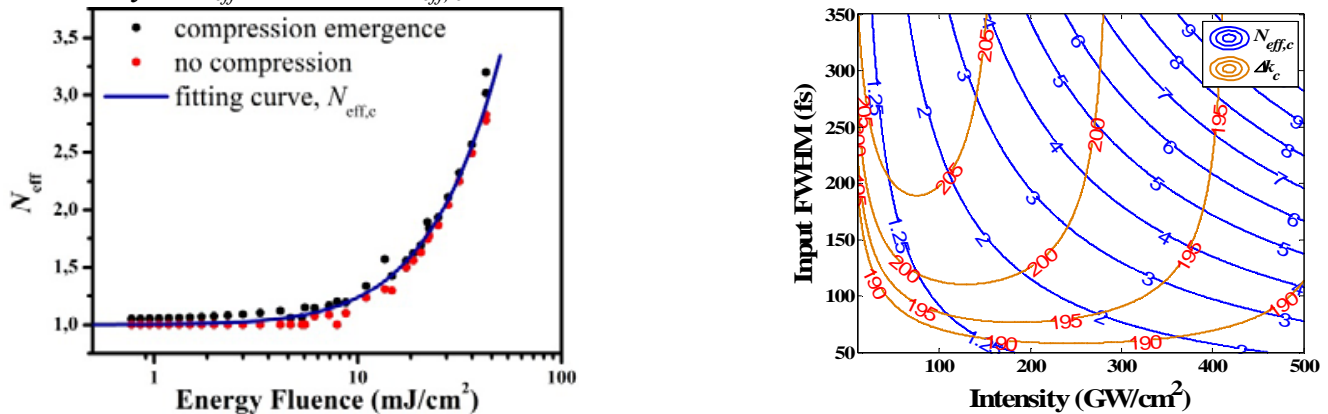

Fig. 2. (a, left) compression boundary $N_{\text {eff,c }}$ as a function of energy fluence at $1550 \mathrm{~nm}$, (b, right) the upper limit $\Delta k_{\mathrm{c}}(\Phi)$ distribution as a function of the input FWHM and peak intensity of the launched pulse.

The revision of the compression condition is equivalent to a revision on the upper limit of the phase mismatch, called critical phase mismatch $\Delta k_{\mathrm{c}}(\Phi)$. Hence, a more precise compression window can be concluded, i.e. $\Delta k_{s r}<$ $\Delta k_{\text {eff }}<\Delta k_{\mathrm{c}}(\Phi)$. With a wide span of the parameters of a launched pulse, the distribution of $\Delta k_{\mathrm{c}}$ and $N_{\text {eff }, c}$ is shown in Fig. 2(b), which is believed helpful in the design of the QPM structure.

Moreover, we also give the dynamics of $N_{\text {eff }}$ together with $N_{\text {eff, } c}$ during the formation of the soliton pulse, shown in Fig. 1(c). It is obvious that the difference $N_{\text {eff }}-N_{\text {eff, } c}$ at the starting point gives the capacity of the pulse compression, and when the best compressed pulse is formed, this capacity is almost exhausted.

\section{Conclusion}

We numerically investigated cascaded quadratic soliton compression in PPLN. With high energy fluence used to supply a cascaded nonlinearity, a critical soliton number becomes significant. Hence, a general critical soliton number $N_{\text {eff. } c}$ is found through a careful study. Moreover, the soliton number dynamics are shown as the capacity of compression and a best compression corresponds to an exhausted capacity. Finally, we presented a phase-space plot where the critical parameters for optimal pulse compression at 1550nm in PPLN can extracted.

\section{Reference}

[1] J. Moses and F. W. Wise, "Soliton compression in quadratic media : high-energy few-cycle pulses with a frequency-doubling crystal," Optics Letters, vol. 31, pp. 1881-1883, (2006).

[2] M. Bache, O. Bang, J. Moses, and F. W. Wise, "Nonlocal explanation of stationary and nonstationary regimes in cascaded soliton pulse compression," Optics Letters, vol. 32, pp. 2490-2492, (2007).

[3] M. Bache, J. Moses, and F. W. Wise, "Scaling laws for soliton pulse compression by cascaded quadratic nonlinearities," J. Opt. Soc. Am. B, vol. 24, pp. 2752-2762, (2007).

[4] B. B. Zhou, A. Chong, F. W. Wise, and M. Bache, "Few-cycle solitons in short strongly phase-mismatched frequency conversion crystals," submitted, arXiv: 1109.4261, (2011). 